\title{
Interaction Induced Quantum Valley Hall Effect in Graphene
}

\author{
E. C. Marino, ${ }^{1}$ Leandro O. Nascimento, ${ }^{1,2}$ Van Sérgio Alves, ${ }^{1,3}$ and C. Morais Smith ${ }^{2}$ \\ ${ }^{1}$ Instituto de Física, Universidade Federal do Rio de Janeiro, \\ CP 68528, Rio de Janeiro, RJ 21941-972, Brazil \\ ${ }^{2}$ Institute for Theoretical Physics, Centre for Extreme Matter and Emergent Phenomena, \\ Utrecht University, Leuvenlaan 4, 3584CE Utrecht, Netherlands \\ ${ }^{3}$ Faculdade de Física, Universidade Federal do Pará, \\ Avenida Augusto Correa 01, 66075-110 Belém, Pará, Brazil
}

(Received 8 October 2013; revised manuscript received 22 September 2014; published 31 March 2015)

\begin{abstract}
We use pseudo-quantum electrodynamics in order to describe the full electromagnetic interaction of the $p$ electrons in graphene in a consistent $2 \mathrm{D}$ formulation. We first consider the effect of this interaction in the vacuum polarization tensor or, equivalently, in the current correlator. This allows us to obtain the $T \rightarrow 0$ conductivity after a smooth zero-frequency limit is taken in Kubo's formula. Thereby, we obtain the usual expression for the minimal conductivity plus corrections due to the interaction that bring it closer to the experimental value. We then predict the onset of an interaction-driven spontaneous quantum valley Hall effect below an activation temperature of the order of $2 \mathrm{~K}$. The transverse (Hall) valley conductivity is evaluated exactly and shown to coincide with the one in the usual quantum Hall effect. Finally, by considering the effects of pseudo-quantum electrodynamics, we show that the electron self-energy is such that a set of $P$ - and $T$-symmetric gapped electron energy eigenstates are dynamically generated, in association with the quantum valley Hall effect.
\end{abstract}

DOI: 10.1103/PhysRevX.5.011040

\section{INTRODUCTION}

The experimental realization of graphene has opened the fascinating possibility of observing in a condensed matter system a number of interesting effects previously considered to occur exclusively in relativistic particle physics. The Klein paradox [1] and the Zitterbewegung [2] are well-known examples. Graphene is also the first concrete realization of the Dirac sea, the concept that led Dirac to predict the existence of antimatter. Indeed, Schwinger's effect of pair creation out of the vacuum by an electric field is expected to occur in this material, thus providing another beautiful connection between condensed matter and particle physics [3].

Graphene exhibits quite a few unconventional transport phenomena. These include an anomalous integer quantum Hall effect (QHE) [4] and a puzzling finite ("minimal") dc conductivity at half filling [5], even in the absence of any dissipation and with a zero density of states. The theoretical determination of the zero-frequency limit of the optical conductivity and its dependence on interactions is still a challenge [6], in part due to the ambiguities associated with the $\omega \rightarrow 0$ limit in Kubo's formula. Attempts to include the effect of interactions in these calculations

Published by the American Physical Society under the terms of the Creative Commons Attribution 3.0 License. Further distribution of this work must maintain attribution to the author(s) and the published article's title, journal citation, and DOI.
Subject Areas: Condensed Matter Physics, Graphene

were recently made [7]; however, this effect disappears in the limit $\omega \rightarrow 0$.

Nevertheless, optical conductivity measurements [5] yielded results that in the dc limit are in agreement with earlier theoretical calculations in the approximation of noninteracting electrons, in the $T \rightarrow 0$ limit, namely, $\sigma_{0}=(\pi / 2) e^{2} / h[8,9]$. Analogously, the integer QHE [4] has been understood in terms of relativistic Landau levels occupied by noninteracting electrons, similarly to the results for GaAs [10,11].

The unexpected validity of the single-particle description has raised the question of how relevant the electronic interactions in graphene are, leading to a vivid debate in the community. Nonetheless, the recent measurement [12] of the renormalization of the Fermi velocity [13] is an indication that interactions should be important. The direct measurement of the dc conductivity [14], which yielded a result that is in disagreement with the theoretical calculation in the absence of interactions, provided additional evidence for the relevance of these. The experimental observation of the fractional QHE in ultraclean samples subject to a perpendicular magnetic field has closed the debate, undeniably demonstrating that the electronic interactions are indeed important, at least for a certain energy (temperature) scale [15-18].

Another intriguing transport property that has been investigated in graphene is the possibility of observing a quantized transverse (Hall) conductivity under unconventional circumstances. First, Haldane has shown that the 
sufficient condition for the existence of the integer QHE is a broken time-reversal symmetry (TRS) and not a net magnetic field, as was previously supposed [19]. Later, even more unexpected results emerged, such as the experimental observation of the integer QHE at room temperature [20] and the proposal for the existence of a quantum spin Hall effect in the presence of a sizable spin-orbit coupling in a system that preserves TRS [21].

Most of the previous approaches, however, rely on a single-particle description, and the role of interactions, as well as the proper theoretical framework to include them, has often been neglected.

Several studies were then performed describing the electronic interaction by means of a static Coulomb potential $[7,22,23]$. These produced very interesting results for $\omega \neq 0$. Yet, this procedure implicitly introduces, from the very beginning, a zero-frequency limit in the current correlator in the Kubo formula, whereas this limit should be taken at the very end [24]. This fact may be responsible for the absence of corrections due to the interaction to the conductivity in the limit $\omega \rightarrow 0$.

From first principles, however, the relevant electronic interaction in graphene is the full electromagnetic interaction described by the minimal coupling of the electronic current to the $\mathrm{U}(1)$ electromagnetic gauge field. This, however, is not easily incorporated in the model because the electrons in graphene are confined to a plane and therefore require a $2 \mathrm{D}$ description, whereas the electromagnetic field is 3D. Should we use Maxwell electrodynamics in 2D for describing the interaction of the electrons in graphene, we would get a wrong result (for instance, the electrostatic potential would be $-\ln r$ instead of the correct $1 / r)$. The solution for this problem consists in the use of a full 2D U(1) gauge field theory, which describes, within the 2D framework, the full physics contained in the 3D Maxwell theory. Such 2D theory was derived in Ref. [25] in the static limit. Subsequently, a full dynamical derivation was provided [26] and the theory was called pseudo-quantum electrodynamics (PQED) (in part because it involves the so-called pseudodifferential operators) [26-28].

In this paper, we employ PQED in order to describe the electronic interactions in graphene and explore some of the consequences of these. We first determine the corrections to the minimal conductivity produced by such interactions, thus obtaining a value at $T=0, \omega \rightarrow 0$, which is the closest to the measured experimental one [14]. We then evaluate the effects of PQED in the valley conductivity and show that, below an activation temperature $T^{*}$, it exhibits a nonzero transverse component, which is quantized in the same way as in the usual QHE. This effect is dynamically generated in graphene, when the full electromagnetic interaction is completely taken into account. In this case, we show that the individual valley contribution to the conductivity contains a $P, T$-violating transverse (Hall) component, which has opposite sign for each valley and consequently leads to a quantum valley Hall effect (QVHE), rather than to the usual QHE. Then, we investigate the Schwinger-Dyson equation for the electron selfenergy in PQED and show that the latter satisfies a differential equation, which has solutions that shift the poles of the electron propagator to gapped energy states when the interaction coupling is larger than a certain critical value. The temperature scale is set by the gap: thermal activation will destroy the plateaus for temperatures larger than $T^{*}$, which corresponds to the gap.

All of the phenomena described here occur only within an $\mathrm{SU}(2)$ description of graphene, which is valid when there is no backscattering connecting the different valleys. We show that the use of PQED, contrary to other attempts to describe the electronic interactions in graphene, yields a current correlator that renders Kubo's formula free from any ambiguities. We also show that our results hold true when the fact that the Fermi velocity is different from the speed of light is taken into account.

The outline of this paper is the following: in Sec. II, we introduce our model within the PQED approach, in Sec. III, we calculate the current-current correlator, which is then used in Sec. IV to derive the conductivity for $T \rightarrow 0$ and $\omega \rightarrow 0$ using the Kubo formula. The fact that $v_{F} \neq c$ is explicitly used in this section. In Sec. V, we show the emergence of a QVHE driven by the interaction, and in Sec. VI, we evaluate the corresponding gaps, which are dynamically generated. In Sec. VII, we present our summary and outlook. We also include two appendixes, where we examine our results in the light of the Vafa-Witten theorem (see Appendix A) and discuss the nonrelativistic limit of Dirac equation (see Appendix B).

\section{MODEL}

The $p$ electrons of the carbon atoms in the honeycomb lattice of graphene are usually described as 4-component massless Dirac fermions, each component corresponding to the two sublattices $(A$ and $B$ ) and the two inequivalent valleys $\left(K\right.$ and $\left.K^{\prime}\right)$. If we neglect backscattering between the valleys, however, an equivalent description would consist of two massless 2-component Dirac fermion fields. We assume that these Dirac electrons will interact through the electromagnetic interaction, which in 2D is described by PQED [26]. The corresponding Lagrangian reads

$$
\mathcal{L}=\frac{1}{4} F_{\mu \nu}\left[\frac{2}{\sqrt{-\square}}\right] F^{\mu \nu}+i \bar{\psi}_{a} \not \psi_{a}+j^{\mu} A_{\mu},
$$

where

$$
i \not \partial=i \gamma^{0} \partial_{0}+i v_{F} \gamma^{i} \partial_{i}
$$

and 


$$
j^{\mu}=e \bar{\psi}_{a} \gamma^{\mu} \psi_{a}=e\left(\bar{\psi}_{a} \gamma^{0} \psi_{a}, v_{F} \bar{\psi}_{a} \gamma^{i} \psi_{a}\right)
$$

$\psi_{a}$ is a 2-component Dirac field, $\bar{\psi}_{a}=\psi_{a}^{\dagger} \gamma^{0}$ is its adjoint, $F_{\mu \nu}$ is the usual field intensity tensor of the U(1) gauge field $A_{\mu}$, which intermediates the electromagnetic interaction in 2D (pseudoelectromagnetic field), $\gamma^{\mu}$ are rank-2 Dirac matrices, and $a=1, \ldots, N_{f}$ is a flavor index, specifying the spin component and the valley to which the electron belongs. The coupling constant $e^{2}=4 \pi \alpha$ is conveniently written in terms of $\alpha$, the fine-structure constant in natural units. Observe that the two valleys $\left(K\right.$ and $\left.K^{\prime}\right)$ are related by TRS. In spite of the fact that we are using 2-component Dirac spinors, however, we do not break TRS ab initio because we are summing over the two species (as we may infer from the physical value of $N_{f}$ ). An SU(4) version of this model has been recently used to study dynamical gap generation and chiral symmetry breaking in graphene [29].

Because of the linear dependence of the Dirac Lagrangian on $p=\left(p_{0}, \mathbf{p}\right)$, it follows that all dependence on $v_{F}$ will appear in the form $v_{F} \mathbf{p}$. In an analogous way, the current changes as $j_{\mu}=\left(j_{0}, j_{i}\right) \rightarrow j_{\mu}=\left(j_{0}, v_{F} j_{i}\right)$ and the current correlation function that we calculate is actually $v_{F}^{2}\langle j j\rangle$. Since the natural velocity appearing in the gauge field sector is that of light $c$, whereas the one occurring in the electronic sector is the Fermi velocity $v_{F}$, Lorentz invariance is broken.

\section{CURRENT-CURRENT CORRELATION FUNCTION}

We determine the $\omega \rightarrow 0$ limit of the optical conductivity in graphene by using the Kubo formula, which describes the linear response to a static external electric field. In real time, it is given by

$$
\sigma^{i k}=\lim _{\omega \rightarrow 0, \mathbf{p} \rightarrow 0} \frac{i\left\langle j^{i} j^{k}\right\rangle}{\omega},
$$

where the current correlation function is meant to contain only one-particle irreducible (1PI) diagrams [24].

The current correlator is most conveniently obtained from the corresponding generating functional. Starting from the generating functional of arbitrary correlators,

$$
\mathcal{Z}[J]=\mathcal{N} \int D A_{\mu} D \bar{\psi} D \psi e^{-\int d^{3} x\left(\mathcal{L}+e j^{\mu} J_{\mu}\right)},
$$

where $J$ is a vector functional variable and $\mathcal{N}=\mathcal{N}_{A} \mathcal{N}_{\psi}$ are constants chosen in such a way that $\mathcal{Z}[0]=1$, we have that the generator of connected correlation functions is given by

$$
W[J]=-\ln \mathcal{Z}[J] .
$$

The generating functional of 1PI correlation functions is then obtained by the following Legendre transformation:

$$
\Gamma\left[A_{c}^{\mu}\right]=\int d^{3} x J_{\mu}(x) A_{c}^{\mu}(x)-W[J],
$$

where

$$
A_{c}^{\mu}(x)=\frac{\delta W[J]}{\delta J_{\mu}(x)}
$$

Thus, the current-current correlation function that is needed for the Kubo formula can be obtained by taking the second derivative of the generating functional,

$$
\left\langle j_{\mu} j_{\nu}\right\rangle=\left.\frac{1}{e^{2}} \frac{\delta^{2}}{\delta A_{c}^{\mu} \delta A_{c}^{\nu}} \Gamma\left[A_{c}\right]\right|_{A_{c}=0} .
$$

It turns out, however, that the above expression is nothing but the $A_{\mu}$ field self-energy $\Pi_{\mu \nu}$, also known as the vacuum polarization tensor, which is given by

$$
G_{\mu \nu}^{-1}-G_{0, \mu \nu}^{-1}=-e^{2} \Pi_{\mu \nu},
$$

where $G$ is the exact $A_{\mu}$ field Euclidean propagator and $G_{0}$ is the free one,

$$
G_{0, \mu \nu}=\frac{1}{2 \sqrt{p^{2}}} P_{\mu \nu} .
$$

In this expression, $P_{\mu \nu}=\delta_{\mu \nu}-p_{\mu} p_{\nu} / p^{2}$, and $p^{2}=p_{3}^{2}+\mathbf{p}^{2}$, where $p_{3}$ is the third component of the energy-momentum vector in the Euclidean space. We, therefore, come to the conclusion that

$$
\left\langle j_{\mu} j_{\nu}\right\rangle_{1 \mathrm{PI}}=\Pi_{\mu \nu} .
$$

$\Pi^{\mu \nu}$ has been calculated up to the order of two loops in PQED, for the case of 2-component fermions. The Euclidean one-loop contribution for a single massless fermion, which is the same for QED in three dimensions (QED3), is [30]

$$
\Pi_{\mu \nu}^{(1)}(p)=A(p) P_{\mu \nu}+B \epsilon_{\mu \nu \alpha} p^{\alpha},
$$

where $A(p)=-\sqrt{p^{2}} / 16$ and $B=(1 / 2 \pi)(n+1 / 2)$, with $n$ integer. Note the occurrence of a $P, T$-breaking term, which is topological and, according to the Coleman-Hill theorem [31], has no higher-order corrections. Even though this result is derived for QED3, it also holds for PQED because it involves only fermion internal lines.

The two-loops correction is exclusive of PQED and was calculated in Ref. [32] for a single massless fermion, yielding

$$
\Pi_{\mu \nu}^{(2)}(p)=-\frac{\sqrt{p^{2}}}{16} C_{\alpha} \alpha_{g} P_{\mu \nu}
$$


where $C_{\alpha}=\left(92-9 \pi^{2}\right) / 18 \pi \approx 0.056$ and $\alpha_{g} \approx 300 / 137=$ 2.189. It follows that $C_{\alpha} \alpha_{g}<1$; hence, the perturbation expansion is justified. Indeed, there is no correction for the $B$ term.

According to Eq. (12), the irreducible current-current correlation function is given by

$$
\left\langle j^{\mu} j^{\nu}\right\rangle=j_{1}(p) P^{\mu \nu}+j_{2} \epsilon^{\mu \nu \alpha} p_{\alpha}
$$

with

$$
j_{1}(p)=-N_{f} A(p)\left[1+C_{\alpha} \alpha_{g}+O\left(e^{4}\right)\right]
$$

and

$$
j_{2}=-N_{f} B
$$

where $N_{f}$ arose from the sum over all fermions.

\section{CONDUCTIVITY FOR $T=0, \omega \rightarrow 0$}

The optical conductivity in the $T=0, \omega \rightarrow 0$ limit can be derived, within the linear response regime, from Kubo's formula, which for real time is

$$
\sigma^{i k}=\lim _{\omega \rightarrow 0, \mathbf{p} \rightarrow 0} \frac{i\left\langle j^{i} j^{k}\right\rangle_{\mathrm{ret}}}{\omega}=\sigma_{x x} \delta^{i k}+\sigma_{x y} \epsilon^{i k} .
$$

Now, when evaluating the current correlation function given by Eqs. (12)-(14), we must replace $\gamma_{i} \rightarrow v_{F} \gamma_{i}$ in the vertices.

The one-loop result in momentum space is

$$
\begin{gathered}
\Pi^{00}\left(p_{0}, \mathbf{p}\right)=-\frac{1}{16} \frac{\mathbf{p}^{2}}{\sqrt{v_{F}^{2} \mathbf{p}^{2}+p_{0}^{2}}}, \\
\Pi^{i 0}\left(p_{0}, \mathbf{p}\right)=\frac{1}{16} \frac{p^{0} p^{i}}{\sqrt{v_{F}^{2} \mathbf{p}^{2}+p_{0}^{2}}}+\frac{1}{2 \pi}\left(n+\frac{1}{2}\right) \epsilon^{i 0 j} p_{j},
\end{gathered}
$$

and

$$
\begin{aligned}
\Pi^{i j}\left(p_{0}, \mathbf{p}\right)= & -\frac{1}{16}\left[\frac{\delta^{i j}\left(v_{F}^{2} \mathbf{p}^{2}+p_{0}^{2}\right)-v_{F}^{2} p^{i} p^{j}}{\sqrt{v_{F}^{2} \mathbf{p}^{2}+p_{0}^{2}}}\right] \\
& +\frac{1}{2 \pi}\left(n+\frac{1}{2}\right) \epsilon^{i j 0} p_{0} .
\end{aligned}
$$

The generating functional $\mathcal{Z}[J]$ is obtained by performing different Gaussian integrals over $A_{0}$ and $A_{i}$. Then, it is easy to see from Eq. (12) that the current correlator is expressed in terms of $\Pi_{i j}, \Pi_{00}$, and $\Pi_{i 0}$. After taking the limit $\mathbf{p} \rightarrow 0$ in the Kubo formula, we conclude that the only contribution comes from $\Pi_{i j}$, since $\Pi_{00}$ and $\Pi_{i 0}$ vanish in this limit. From Eq. (21), however, we see that all dependence on $v_{F}$ disappears in the limit when the external momentum $\mathbf{p} \rightarrow 0$. Note that the above argument also holds for the two-loops contribution.

The current correlator [Eq. (12)] is proportional to the number of flavors $N_{f}$, consisting of spin $\uparrow, \downarrow$ and valleys $K, K^{\prime}$. We have, therefore, $N_{f}=N_{S}+N_{V}$. The two spin components give identical contributions to Eq. (4); therefore, $N_{S}=2$. We must be careful, however, when summing the contributions from the two valleys $K$ and $K^{\prime}$. For symmetry reasons, it is reasonable to expect that both valleys will contribute identically. Nevertheless, the valleys $K$ and $K^{\prime}$ are related to each other by TRS and, consequently, their contribution will depend on whether this symmetry is spontaneously broken or not. When TRS symmetry is preserved, both valleys clearly give identical contributions and $N_{V}=2$ or $N_{f}=4$.

Indeed, in linear response theory, for each valley, we have

$$
\left\langle 0\left|j^{i}\right| 0\right\rangle_{K}=\frac{i\left\langle 0\left|j_{K}^{i} j_{K}^{j}\right| 0\right\rangle}{\omega} E^{j}
$$

and

$$
\left\langle 0\left|j^{i}\right| 0\right\rangle_{K^{\prime}}=\frac{i\left\langle 0\left|j_{K^{\prime}}^{i} j_{K^{\prime}}^{j}\right| 0\right\rangle}{\omega} E^{j}
$$

where $E^{j}$ is an external electric field. The contribution from the two valleys to the average total current is given by $\left\langle 0\left|j_{K}^{i}+j_{K^{\prime}}^{i}\right| 0\right\rangle$. According to the result above, this can be expressed as

$$
\begin{aligned}
& \left\langle 0\left|j^{i}\right| 0\right\rangle_{K}+\left\langle 0\left|j^{i}\right| 0\right\rangle_{K^{\prime}} \\
& =\left\{\frac{i\left\langle 0\left|j_{K}^{i} j_{K}^{j}\right| 0\right\rangle}{\omega}+\frac{i\left\langle 0\left|j_{K}^{i} j_{K}^{j}\right| 0\right\rangle_{T}}{\omega}\right\} E^{j} .
\end{aligned}
$$

Therefore, when the TRS is not spontaneously broken, the sum of the contributions from the two valleys to the conductivity is

$$
\sigma^{i k}=\lim _{\omega \rightarrow 0, \mathbf{p} \rightarrow 0}\left\{\frac{i\left\langle j^{i} j^{k}\right\rangle}{\omega}+\frac{i\left\langle j^{i} j^{k}\right\rangle^{T}}{\omega}\right\}
$$

where $\langle j j\rangle^{T}$ is the time-reversed correlator and the sum over spins is assumed to have been done, namely, at this level $N_{f}=N_{S}=2$.

Now, observe that, according to Eqs. (16) and (17), $j_{2}$ is a constant, whereas $j_{1}$ is a function of $\mathbf{p}^{2}+p_{3}^{2}$ in Euclidean space. When we go back to the real time, we must analytically continue $p_{3}$ to the imaginary axis. Hence, $j_{1}$ becomes a function of $\mathbf{p}^{2}+\left(i p_{0}\right)^{2}$. This is invariant under time reversal $\left(i \rightarrow-i, p_{0} \rightarrow p_{0}\right.$ and $\left.\mathbf{p} \rightarrow-\mathbf{p}\right)$ and, consequently, so is $j_{1}$. 
In the limit $\mathbf{p} \rightarrow 0$, the current correlator and its timereversed version are given, respectively, by expressions of the form

$$
\left\langle j^{i} j^{k}\right\rangle=j_{1}\left(\left(i p_{0}\right)^{2}\right) \delta^{i k}+j_{2} \epsilon^{i k}\left(i p_{0}\right),
$$

and

$$
\left\langle j^{i} j^{k}\right\rangle^{T}=j_{1}\left(\left(-i p_{0}\right)^{2}\right) \delta^{i k}+j_{2} \epsilon^{i k}\left(-i p_{0}\right) .
$$

The first term is clearly invariant, since $\left(i p_{0}\right)^{2}=$ $\left(-i p_{0}\right)^{2}$. The second term, conversely, is clearly noninvariant and derives from the anomalous part of the vacuum polarization tensor, which is generated by vacuum fluctuations. The $p_{0}$ variable above, in the unit system that we are using, must be identified with the frequency $\omega$ in the Kubo formula [Eq. (4)].

We now take the limit $\omega \rightarrow 0$ in the optical conductivity. It is worth mentioning that this limit in the Kubo formula can be taken unambiguously when PQED is used to describe the interactions, unlike the usual QED3. This occurs due to the peculiar structure of the gauge field propagator of the theory, which produces a linear $\omega$ dependence in the current correlator for $\mathbf{p} \rightarrow 0$, which will cancel the $\omega$ in the denominator in the Kubo formula. Also, we can understand why any attempt to use a static Coulomb $1 / r$ interaction for determining the corrections to the $\omega \rightarrow 0$ limit of the optical conductivity does not succeed. When we follow this procedure, we are, from the very outset, implicitly making $\omega=0$, thus reversing the correct order in which the limits should be taken in Kubo's formula.

Using Eqs. (26) and (27), we see that the conductivity has the general form

$$
\sigma^{i k}=\sigma^{x x} \delta^{i k}+\sigma^{x y} \epsilon^{i k} .
$$

Inserting Eqs. (26) and (27) into Eq. (25), we find that for an unbroken TRS phase, only the longitudinal part survives. The two valleys contribute in the same way; hence, $N_{V}=2$ or $N_{f}=4$. The $p_{0}$ dependence cancels out, and we can take the zero-frequency limit without hurdles. Using Eq. (25), we obtain

$$
\sigma^{x x}=\left(\frac{\pi}{2} \frac{e^{2}}{h}\right)\left[1+\left(\frac{92-9 \pi^{2}}{18 \pi}\right) \alpha_{g}+\mathcal{O}\left(e^{4}\right)\right]
$$

and

$$
\sigma^{x y}=0 .
$$

To put our results into the literature context, notice that the collision-dominated limit $\hbar \omega \ll k_{B} T$ was investigated in Ref. [33] using the quantum Boltzmann equation. Here, we study the optical conductivity in the regime $\omega \gg k_{B} T / \hbar$ (since we start from $T=0$ from the beginning and then take the limit $\omega \rightarrow 0$ ) and determine the correction provided by the full electromagnetic interaction to the noninteracting value $\sigma_{0}=\pi e^{2} / 2 h$. To the best of our knowledge, the value we find for the conductivity in this limit, namely, $\sigma_{x x}=1.76 e^{2} / h$, is the closest to the experimental result for the conductivity extrapolated to zero temperature, namely, $\sigma_{x x}=2.16 e^{2} / h$ [14]. In spite of this, our result is yet somewhat far from the experimental result. Further investigations including disorder, for instance, may be required to achieve a better agreement between theory and experiments.

\section{QUANTUM VALLEY HALL EFFECT}

The average valley current is defined by

$$
\left\langle J_{V}^{i}\right\rangle=\left\langle 0\left|j_{K}^{i}\right| 0\right\rangle-\left\langle 0\left|j_{K^{\prime}}^{i}\right| 0\right\rangle .
$$

It vanishes whenever the two valleys contribute the same amount to the electric current. From Eq. (31), it is clear that

$$
\left\langle J_{V}^{i}\right\rangle=\left\{\frac{i\left\langle 0\left|j_{K}^{i} j_{K}^{j}\right| 0\right\rangle}{\omega}-\frac{i\left\langle 0\left|j_{K}^{i} j_{K}^{j}\right| 0\right\rangle_{T}}{\omega}\right\} E^{j} .
$$

We can, therefore, define the zero-frequency limit of the "optical valley conductivity," which is given by

$$
\sigma_{V}^{i k}=\lim _{\omega \rightarrow 0, \mathbf{p} \rightarrow 0}\left\{\frac{i\left\langle j^{i} j^{k}\right\rangle}{\omega}-\frac{i\left\langle j^{i} j^{k}\right\rangle^{T}}{\omega}\right\},
$$

where the sum over spins is assumed to have been done. One immediately concludes that the longitudinal parts cancel, whereas the transverse component survives. The valley conductivity, therefore, is given by

$$
\sigma_{V}^{x y}=4\left(n+\frac{1}{2}\right) \frac{e^{2}}{h},
$$

for $n=$ integer. The longitudinal component, conversely, vanishes:

$$
\sigma_{V}^{x x}=0 .
$$

The above result is exact, as a consequence of the Coleman-Hill theorem. The existence of a transverse valley conductivity characterizes the occurrence of a QVHE. It is caused, ultimately, by the presence of the anomalous $P, T$-violating term appearing in the vacuum polarization tensor or, equivalently, in the current correlator. In the next section, we show that this effect is predicted to occur for temperatures $T<T^{*}$, where $T^{*}$ is a temperature above which the effect is destroyed by thermal activation.

The valley Hall effect has been earlier predicted to occur in graphene systems subject to a staggered sublattice potential that breaks inversion symmetry [34,35], or to strained graphene, where according to recent experiments 
pseudomagnetic fields oppositely oriented in the valleys can be as large as $300 \mathrm{~T}$ [36]. In addition, a fractional valley Hall effect was proposed to arise in artificial graphene systems, by fine-tuning the short-range part of interactions [37]. Note that here no symmetry is broken a priori and no fine-tuning of model parameters is required to generate the QVHE. A similar TRS breaking was recently proposed to occur for bilayer graphene in the presence of static Coulomb interactions, when fluctuations are taken into account [38].

The anomalous terms found here are related to electron masses that are dynamically generated. These, however, appear in pairs of opposite signs and, for an even number of flavors, cancel when summed, according to the Vafa-Witten theorem [39]. Consequently, there is no overall $P, T$ violation, and for this reason, the QHE does not occur. The existence of individually violating terms, nevertheless, is sufficient to produce a QVHE. This is the central result of this work.

\section{DYNAMICALLY GENERATED DISCRETE ENERGY STATES AND $T^{*}$}

Recently, it has been shown that the model described by Eq. (1) dynamically generates a gap in the SU(4) case due to a breaking of the chiral symmetry [29]. Here, we investigate the $\mathrm{SU}(2)$ case and show that an infinite sequence of discrete energy eigenstates is dynamically generated.

For the 2-component Dirac fields considered here, the associated gap generation breaks the parity and timereversal symmetries instead of the chiral one. The generation of this set of eigenstates, which is a consequence of the interactions, is therefore another manifestation of the dynamical symmetry breaking found in the vacuum polarization tensor, which has led to the spontaneous QHE for each valley below $T^{*}$ in Sec. V. The result is obtained by a nonperturbative solution of the Schwinger-Dyson equation [40],

$$
S_{F}^{-1}(p)=S_{0 F}^{-1}(p)-\Sigma(p)
$$

where $S_{0 F}$ and $S_{F}$ are, respectively, the free- and interactingelectron propagators and $\Sigma(p)$ is the electron self-energy, which is given by

$$
\Sigma(p)=e^{2} \int \frac{d^{3} k}{(2 \pi)^{3}} \gamma^{\mu} S_{F}(k) \gamma^{\nu} G_{\mu \nu}(p-k),
$$

where $G_{\mu \nu}$ is the full field propagator of the gauge field. For the sake of simplicity, we first consider $v_{F}=c$ and then modify the calculation for $v_{F} \neq c$.
By making a Taylor expansion around $\epsilon$,

$\Sigma(p)=\Sigma(p=\epsilon)+\left.\left(\gamma^{\mu} p_{\mu}-\epsilon\right) \frac{\partial \Sigma(p)}{\partial p}\right|_{p=\epsilon}+\cdots$,

and imposing

$$
\Sigma(p=\epsilon)=\epsilon,
$$

we write the full fermion propagator as

$$
\begin{aligned}
S_{F}(p) & =\frac{1}{\gamma^{\mu} p_{\mu}-\Sigma(p)} \\
& =\frac{1}{\left(\gamma^{\mu} p_{\mu}-\epsilon\right)\left(1-\left.\frac{\partial \Sigma(p)}{\partial p}\right|_{p=\epsilon}+\cdots\right)} \\
& =\frac{\gamma^{\mu} p_{\mu}+\epsilon}{\left(p^{2}-\epsilon^{2}\right)\left(1-\left.\frac{\partial \Sigma(p)}{\partial p}\right|_{p=\epsilon}+\cdots\right)} .
\end{aligned}
$$

We see that $\epsilon$ is the pole of the full physical electron propagator at zero momentum, being, therefore, an eigenenergy. Using an $e^{2}$ expansion, the gauge field propagator can be written as

$$
G_{\mu \nu} \approx \frac{1}{\sqrt{p^{2}}\left(2+\frac{\lambda}{16}\right)} P_{\mu \nu}
$$

where $\lambda=e^{2} N_{f}=4 \pi \alpha N_{f}$.

Inserting Eq. (36) and Eq. (41) into Eq. (37), we obtain the integral equation

$$
\Sigma_{1}(p)=\frac{2 \lambda}{N_{f}} \int \frac{d^{3} k}{(2 \pi)^{3}} \frac{\Sigma_{1}(k)}{k^{2}+\Sigma_{1}^{2}(k)} \frac{1}{\sqrt{(p-k)^{2}}\left(2+\frac{\lambda}{16}\right)},
$$

where $2 \Sigma_{1}(p)=\operatorname{tr} \Sigma(p)$, with tr denoting the trace over Dirac matrices. Introducing an ultraviolet cutoff $\Lambda$, we can transform the integral equation (42) into a differential equation (Euler's equation),

$$
\frac{d}{d p}\left(p^{2} \frac{d \Sigma_{1}(p)}{d p}\right)+\frac{N_{c}}{4 N_{f}} \Sigma_{1}(p)=0
$$

where

$$
N_{c}=\frac{4 \lambda}{\pi^{2}\left(2+\frac{\lambda}{16}\right)} .
$$

The self-energy also obeys

$$
\lim _{p \rightarrow \Lambda}\left(2 p \frac{d \Sigma_{1}(p)}{d p}+\Sigma_{1}(p)\right)=0
$$

and 


$$
\lim _{p \rightarrow 0} p^{2} \frac{d \Sigma_{1}(p)}{d p}=0
$$

representing the ultraviolet and infrared boundary conditions, respectively.

The solutions of Euler's differential equation are

$$
\Sigma_{1}(p)=\tilde{C} p^{a_{+}}+\tilde{D} p^{a_{-}},
$$

where $a_{ \pm}=-1 / 2 \pm 1 / 2 \sqrt{1-N_{c} / N_{f}}$ and $\tilde{C}$ and $\tilde{D}$ are constants. The solution Eq. (47) can, without loss of generality, be rewritten as

$\Sigma_{1}(p)=\frac{(C+D)}{\sqrt{p}} \cos \left(\gamma \ln \frac{p}{\bar{\Lambda}}\right)+i \frac{(C-D)}{\sqrt{p}} \sin \left(\gamma \ln \frac{p}{\bar{\Lambda}}\right)$,

where $C=\tilde{C} \bar{\Lambda}^{i \gamma}, D=\tilde{D} \bar{\Lambda}^{-i \gamma}$, and the constant $\gamma$ is given by $\gamma=(1 / 2) \sqrt{N_{c} / N_{f}-1}$. For $N_{f}=4$, it reads

$$
\gamma=\frac{2}{\sqrt{\pi}} \sqrt{\left(\frac{\alpha}{2+\pi \alpha}\right)-\left(\frac{\alpha_{c}}{2+\pi \alpha_{c}}\right)},
$$

where the critical coupling

$$
\alpha_{c}=\frac{2 \pi}{16-\pi^{2}} \simeq 1.02
$$

Observe that $\gamma$ is real in the range of couplings such that $\alpha>\alpha_{c}$, because the quantity between parentheses in Eq. (49) is monotonically increasing.

Insertion of Eq. (48) into the boundary condition [Eq. (45)] provides us with the constraints on the values of $C$ and $D$, namely,

$$
\begin{gathered}
\lim _{p \rightarrow \Lambda} \frac{2 \gamma}{\sqrt{p}}\left[(C+D) \sin \left(\gamma \ln \frac{p}{\bar{\Lambda}}\right)\right. \\
\left.+i(D-C) \cos \left(\gamma \ln \frac{p}{\bar{\Lambda}}\right)\right]=0 .
\end{gathered}
$$

There are two possible solutions that obey the constraint: either $C=D$ and $\sin [\gamma \ln (\Lambda / \bar{\Lambda})]=0$ or $C=-D$ and $\cos [\gamma \ln (\Lambda / \bar{\Lambda})]=0$.

By assuming $2 C=\Lambda^{3 / 2}$ to regularize the constraint, we rewrite Eq. (48) as

$$
\begin{array}{ll}
\Sigma_{1}(p)=\frac{\Lambda^{3 / 2}}{\sqrt{-p}} \sin \left(\gamma \ln \frac{p}{\bar{\Lambda}}\right), & C=-D, \\
\Sigma_{1}(p)=\frac{\Lambda^{3 / 2}}{\sqrt{p}} \cos \left(\gamma \ln \frac{p}{\bar{\Lambda}}\right), & C=D,
\end{array}
$$

where the constant $\bar{\Lambda}$ can be obtained from Eq. (45), namely,

$$
\begin{gathered}
\bar{\Lambda}=\Lambda \exp \left[-\frac{(2 l+1) \pi}{2 \gamma}\right], \quad C=-D, \\
\bar{\Lambda}=\Lambda \exp \left[-\frac{k \pi}{\gamma}\right], \quad C=D,
\end{gathered}
$$

with $k$ and $l$ integers. Now, we choose these integers to have a value as small as possible, but in a way to guarantee that $\Lambda \geq \bar{\Lambda}$. This choice fixes $l=k=0$.

In order to obtain the physical eigenenergies $\epsilon$, we must solve Eq. (39). Using Eqs. (52) and (53) for the self-energy and taking the trace of Eq. (39), we have

$$
\begin{gathered}
\epsilon=-\frac{\Lambda^{3 / 2}}{\sqrt{-\epsilon}} \cos \left(\gamma \ln \frac{|\epsilon|}{\Lambda}\right), \quad \epsilon<0, \\
\epsilon=\frac{\Lambda^{3 / 2}}{\sqrt{\epsilon}} \cos \left(\gamma \ln \frac{|\epsilon|}{\Lambda}\right), \quad \epsilon>0 .
\end{gathered}
$$

We now define

$$
z=-\gamma \ln (|\epsilon| / \Lambda) \quad(z>0)
$$

or, equivalently,

$$
|\epsilon|=\Lambda \exp \left(-\frac{z}{\gamma}\right)
$$

Then, after inserting Eq. (59) into Eqs. (56) and (57), we find that the dimensionless quantity $z(\gamma)$ is given by the solutions of the transcendental equation

$$
\exp \left(-\frac{3 z}{2 \gamma}\right)=\cos z
$$

which holds for both $\epsilon$ positive or negative. Its solutions depend on $\gamma$, which on its turn is determined by the coupling and the number of flavors $N_{f}$. In Fig. 1, we show the graphical solutions of the equation above.

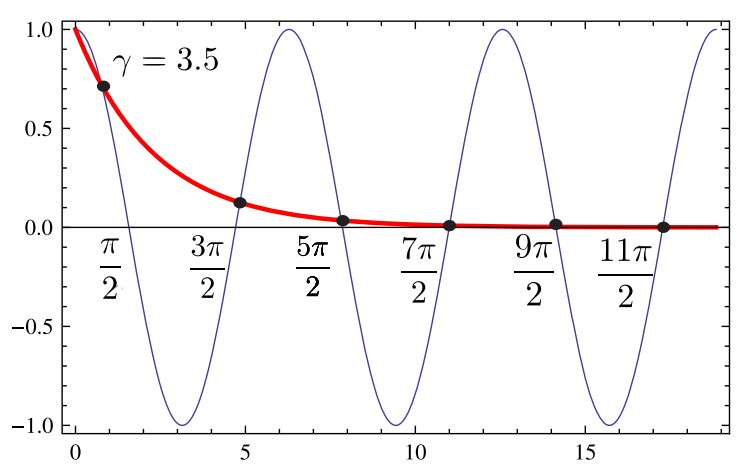

FIG. 1. Graphical representation for solutions of the transcendental equation. The value of $\gamma$ is artificial, to facilitate the visualization. 
We call $z_{n}=Z_{n}, n=0,1,2, \ldots$, the solutions of Eq. (60). It is not difficult to infer, from the graphic representation of the functions in Eq. (60), that

$$
Z_{n}=n \pi+\delta_{n},
$$

where

$$
\begin{array}{ll}
0 \leq \delta_{n} \leq \frac{\pi}{2}, & n=0,2,4, \ldots, \\
\frac{\pi}{2} \leq \delta_{n} \leq \pi, & n=1,3,5, \ldots
\end{array}
$$

For all values of $n, \delta_{n} \rightarrow \pi / 2$ for $n \rightarrow \infty$, whereas $\delta_{n} \rightarrow 0$ ( $n$ even) and $\delta_{n} \rightarrow \pi$ ( $n$ odd) for $\gamma \rightarrow \infty$ (an unphysical limit, in which $N_{f} \rightarrow 0$ ).

The energy levels are then

$$
\epsilon_{n}^{( \pm)}= \pm \Lambda \exp \left\{-\frac{Z_{n}}{\gamma}\right\}
$$

Observe that the negative energy levels increase and the positive ones decrease with $n=0,1,2, \ldots$, in such a way that both of them accumulate in zero for $n \rightarrow \infty$. In the situation when $\gamma \rightarrow 0$, which occurs when $\alpha \rightarrow \alpha_{c}$, all energy levels $\epsilon_{n}^{( \pm)}$collapse to zero, thus destroying the effect. Therefore, $\alpha_{c}$ is a critical quantity for the phenomenon we describe: this will occur only for $\alpha>\alpha_{c}$ for $N_{f}=4$. Since $\gamma$ is small, the lhs of the Eq. (60) tends to zero and the solutions are the zeros of the cosine function, which are $Z_{n} \approx(2 n+1) \pi / 2$.

Since the eigenenergies are zero-momentum poles of the corrected electron propagator, they become dynamically generated electron masses. Notice that all flavors will acquire a mass

$$
M=\epsilon_{0}^{( \pm)} \simeq \pm \Lambda \exp \left\{-\frac{\pi}{2 \gamma}\right\} .
$$

Fixing the renormalization-group invariant gap $M$, we obtain an expression for the renormalized coupling $\alpha(\Lambda)$ as a function of the scale $\Lambda$ :

$$
\frac{\pi}{4} \frac{\sqrt{\pi}}{\sqrt{\left(\frac{\alpha}{2+\pi \alpha}\right)-\left(\frac{\alpha_{c}}{2+\pi \alpha_{c}}\right)}}=\ln \left[\frac{\Lambda}{M}\right] .
$$

Notice that $\alpha_{c}$ is an ultraviolet fixed point.

We may now estimate the activation temperature threshold $T^{*}$ for the observation of the effect we found. This corresponds to the thermal activation energy $E_{a}$, which is such that $E_{a} \geq M$, where the gap $M$ is determined from Eq. (65). According to the Arrhenius law [41],

$$
\exp \left\{-\frac{E_{a}}{k_{B} T^{*}}\right\}=\frac{N}{N_{0}},
$$

where the rhs expresses the ratio of successful activation events, which usually is of the order of $10^{-14}$. We find, therefore,

$$
T^{*} \simeq \frac{M}{14 k_{B} \ln 10} .
$$

Observe that $T^{*} \rightarrow 0$ as $\alpha \rightarrow \alpha_{c}$. In Fig. 2, we plot $T^{*}$ as a function of the coupling $\alpha$ for $N_{f}=4$. By using the measured value of the coupling for suspended graphene in vacuum, namely, $\alpha \simeq 2.189$, we estimate the upper temperature threshold for observation of the spontaneous QVHE to be of the order of $2 \mathrm{~K}$.

We finally remark that the existence of a one-to-one mapping between the energy bands with the respective gaps and the valley Hall conductivity plateaus, which count the number of edge states, is reminiscent of the bulk-boundary correspondence, known to apply for topological insulators. This holds in spite of the fact that there is no $Z_{2}$ topological invariant associated with the QVHE, because when we integrate over the Brillouin zone, the contributions due to the $K$ and $K^{\prime}$ valleys cancel out.

It is worth emphasizing that the study of dynamical mass generation for electrons in graphene has been investigated in the literature in different contexts, by considering only the static Coulomb interaction with screening effect. In this case, the quantum corrections in the gauge propagator contain only the " 00 " component of the vacuum polarization [42-44]. The influence of the renormalization of the Fermi velocity on the gap equation was investigated in Ref. [44].

We consider now the effects of $v_{F} \neq 1$ and $c \neq 1$ on the self-energy $\Sigma(p)$, on the dynamically generated gaps $\epsilon_{n}$, and on the activation temperature $T^{*}$. Now, the previous argument with the scale transformation cannot be used

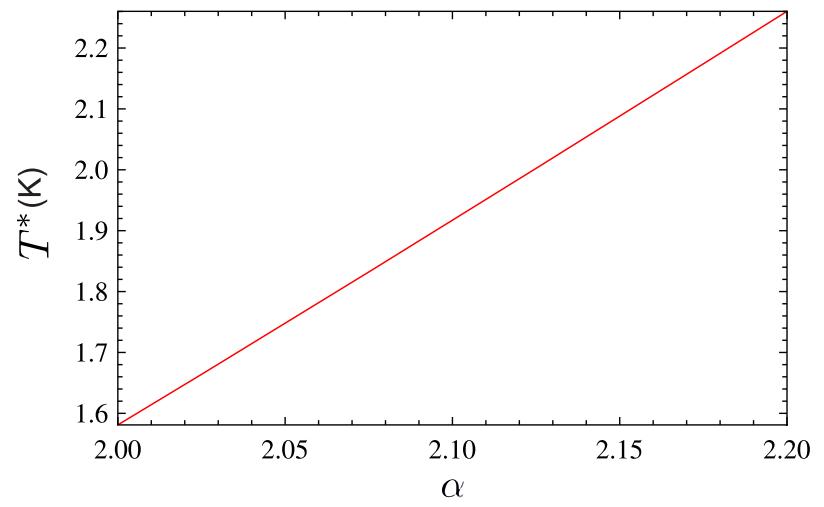

FIG. 2. Activation temperature $T^{*}$ as a function of the coupling $\alpha$ for $N_{f}=4$ and $\Lambda \sim 3.0 \mathrm{eV}$. 
despite the fact that the propagators are massless, because each of them contains a different velocity.

In the relativistic case $\left(v_{F}=c=1\right)$, the self-energy is a function $\Sigma\left(\sqrt{p_{0}^{2}+\mathbf{p}^{2}}\right)$. When we reinstate the physical values of $v_{F}$ and $c$, it happens that the self-energy becomes a function $\Sigma\left(f_{1}\left(v_{F}, c\right) p_{0}, f_{2}\left(v_{F}, c\right) \mathbf{p}\right)$ [45], where the coefficient $f_{1}\left(v_{F}, c\right)$ is dimensionless, whereas $f_{2}\left(v_{F}, c\right)$ has dimension of velocity. Note that $p_{0}$ has dimension of energy when we use the physical units.

We are interested in the dynamically generated gap, i.e., the mass spectrum; hence, we need to evaluate $\Sigma\left(f_{1} p_{0}, f_{2} \mathbf{p}=0\right)$ only. Therefore, we make the Taylor expansion of the self-energy in the variable $p_{0}$ around the gap $\epsilon$, namely,

$\Sigma\left(f_{1} p_{0}\right)=\Sigma\left(f_{1} \epsilon\right)+\left.\left(\gamma^{0} p_{0}-\epsilon\right) \frac{\partial \Sigma\left(f_{1} p_{0}\right)}{\partial p_{0}}\right|_{p_{0}=\epsilon}+\cdots$.

Now, we must impose the condition

$$
\Sigma\left(f_{1} \epsilon\right)=\epsilon
$$

instead of Eq. (39).

The full fermion propagator at zero momentum becomes

$$
\begin{aligned}
S_{F}\left(p_{0}, \mathbf{p}=0\right) & =\frac{1}{\gamma^{0} p_{0}-\Sigma\left(f_{1} p_{0}\right)} \\
& =\frac{1}{\left(\gamma^{0} p_{0}-\epsilon\right)\left(1-\left.\frac{\partial \Sigma\left(f_{1} p_{0}\right)}{\partial p_{0}}\right|_{p_{0}=\epsilon}+\cdots\right)} \\
& =\frac{\gamma^{0} p_{0}+\epsilon}{\left(p_{0}^{2}-\epsilon^{2}\right)\left(1-\left.\frac{\partial \Sigma\left(f_{1} p_{0}\right)}{\partial p_{0}}\right|_{p_{0}=\epsilon}+\cdots\right)},
\end{aligned}
$$

and the dynamically generated gap is still $\epsilon$. This is determined by Eq. (69), which yields the solutions

$$
\bar{\epsilon}_{n}^{( \pm)}= \pm \Lambda \exp \left\{-\frac{\bar{Z}_{n}}{\gamma}\right\}
$$

where $\bar{Z}_{n}$ are solutions of the equation

$$
\exp \left(-\frac{3}{2 \gamma} z\right)=f_{1}\left(v_{F}, c\right) \cos z
$$

For physical values of the coupling constant of graphene, $\gamma$ is rather small. It follows that the left-hand side of Eq. (71) is close to zero, as before. Consequently, the solutions of Eq. (71) are effectively given by the zeros of the cosine function, independently of the value of $f_{1}\left(v_{F}, c\right)$. Hence, we conclude that $\bar{Z}_{n}$ coincide with $Z_{n}$ and the dynamically generated gaps $\bar{\epsilon}_{n}^{( \pm)}$are the same as before. This fact implies that our estimate for the activation temperature $T^{*}$ remains unchanged when the physical values of $v_{F}$ and $c$ are used.

\section{SUMMARY AND OUTLOOK}

Experimental and theoretical results suggest that electronic interactions must be important in graphene, at least for a certain temperature range. The observation of the fractional QHE [15-17] is an example of the former, whereas renormalization group calculations, which show an increase of the interaction strength as we lower the temperature [13], is an example of the latter.

We provide a complete and strictly $2 \mathrm{D}$ description of the real electromagnetic interactions occurring among the electrons in graphene, by means of PQED. This allows us to take the zero limits in the Kubo formula in the correct order, thus obtaining the longitudinal conductivity of graphene in the limits $T=0$ and $\omega \rightarrow 0$. Our result yields the "minimal" value plus corrections due to the interaction, which make it, to the best of our knowledge, the closest to the experimental result for the conductivity at $T=0$.

In addition, the interaction dynamically generates, through one-loop vacuum fluctuations, a term that potentially could produce TRS breaking. This induces a transverse (Hall) valley conductivity quantized exactly as if there was an external magnetic field in the QHE, below an activation temperature $T^{*}$. Discrete states corresponding to the Hall plateaus, and analogous to the Landau levels in the usual QHE, are generated as interaction induced renormalized poles of the fully corrected electron propagator at zero momentum. TRS is restored when we sum over all generated states.

The quantization of our valley currents is emergent, exact, and universal, contrarily to the results obtained in the literature for a QVHE driven by inversion symmetry breaking (staggered chemical potential) $[34,35,46]$. Even though our calculations are made at $T=0$, we estimate the upper temperature threshold $T^{*}$ for observing the effect by identifying it with the Arrhenius activation temperature. This follows from the fact that, when the temperature reaches a level such that most of the states would be populated by thermal activation, the plateaus would be washed out.

Let us remark here that, despite the fact of seemingly being nonlocal, $\mathrm{PQED}$ has been proved to respect causality [27] and also unitarity [47].

The easiest way to experimentally observe the phenomenon predicted here is by first implementing a valley filtering in the graphene system, and then performing usual transport measurements of the electronic Hall conductivity. A second possibility is to use light to probe the valley conductivity, since electrons from different valleys are sensitive to the circular polarization of light [46], thus producing a circular dichroism whenever they are spatially separated. During the past years, several setups have been suggested as a way to spatially separate the contribution of 
the different $\left(K, K^{\prime}\right)$ valleys (Ref. [48-51]). In Ref. [48], a valley filter is proposed to be realized by passing an electronic current through graphene nanoconstrictions with zigzag edges. A second alternative is provided by investigating graphene samples with a line defect, as experimentally realized in Ref. [52]. In this case, quasiparticles hitting the line defect nearly perpendicularly will be filtered with almost $100 \%$ valley polarization [49]. Indeed, the line defect fully transmits (reflects) quasiparticles originating from the $K\left(K^{\prime}\right)$ valley, thus separating both valleys in space. The result is then a time-reversal symmetry-broken quantum Hall effect for each valley in each edge of the sample, which should be detectable through usual transport measurements or unpolarized light and exhibit a quantized Hall conductivity of $\sigma_{K}=2(n+1 / 2) e^{2} / h$ at a single edge. We hope that our results will trigger further experiments to observe this fascinating interaction-driven QVHE at low temperatures. This work opens the path to exactly quantized valleytronics in graphene, thus bringing the field a step further in comparison to the noninteracting model studied in silicene [46].

\section{ACKNOWLEDGMENTS}

This work was supported in part by CNPq (Brazil), CAPES (Brazil), FAPERJ (Brazil), The Netherlands Organization for Scientific Research (NWO), and by the Brazilian government project Science Without Borders. The work by C. M. S. is part of the D-ITP consortium, a program of the Netherlands Organisation for Scientific Research (NWO) that is funded by the Dutch Ministry of Education, Culture and Science (OCW). We are grateful to E. Andrei, G.'t Hooft, A. H. Castro Neto, M. Goerbig, L. Fritz, M. Freedman, and V. Juričić for very interesting and stimulating discussions.

\section{APPENDIX A: VAFA-WITTEN THEOREM}

Here, we examine our results in the light of the VafaWitten theorem [39]. We start by reviewing the proof of the theorem. Consider the partition function of a gauge field with a vector minimal coupling to a Dirac field of mass $M$ in Euclidean space. Integration over the Dirac field yields

$$
\mathcal{Z}[\xi]=\mathcal{N} \int D A_{\mu} e^{-S_{\xi}\left[A_{\mu}\right]} \operatorname{Det}[\not D+M]\left[A_{\mu}\right] .
$$

On general grounds, the bosonic part of the action may be decomposed into a $P$-invariant (and $T$-invariant) part $S_{0}$ and a $P$-noninvariant (and $T$-noninvariant) part $i \xi X$, which in Euclidean space is purely imaginary. Indeed, in the expression above $S_{\xi}=S_{0}+i \xi X$ [39], where $\xi$ is a real parameter.

The theorem follows from the fact that, for a real and positive fermionic determinant, evidently, we have a bound $\mathcal{Z}[\xi] \leq \mathcal{Z}[0]$. Defining $\mathcal{Z}[\xi]=e^{-\mathcal{V}[\xi]}$, we have $\mathcal{V}[\xi] \geq \mathcal{V}[0]$.
Hence, the energetically most stable state is the one with $\xi=0$, implying $\langle X\rangle=0$, which means that there is no spontaneous breakdown of $P$ and $T$ symmetries. This completes the proof.

A key ingredient for the demonstration of the VafaWitten theorem is the fact that the fermionic determinant must be real and positive. This is guaranteed by the following lemma: Suppose the anti-Hermitian operator $\not D$ has eigenvalues $i \lambda$;

$$
[\not D+M] \psi=[M+i \lambda] \psi
$$

Since the $\gamma^{5}$ matrix anticommutes with $\not D$, it follows that for each eigenstate $\psi$, there will be another one given by $\gamma^{5} \psi$, with eigenvalue $[M-i \lambda]$. The fermionic determinant, accordingly, will be $\prod_{\lambda}\left[M^{2}+\lambda^{2}\right]$, which is real and positive, thus completing the proof of the lemma.

We now come to the system we are using for describing graphene. A great difference with respect to the framework where the Vafa-Witten theorem has been demonstrated is the fact that there is no $\gamma^{5}$ matrix for 2-component Dirac fermions in two spatial dimensions; hence, the above lemma, which forced the fermionic determinant to be real and positive, does not apply.

The fermionic determinant was actually calculated in Ref. [30] for a single 2-component fermion in twodimensional space and, indeed, it presents a complex phase. This is proportional to a Chern-Simons term, which is not invariant under either $P$ or $T$, and the proportionality factor is fixed and nonvanishing. In this case, the theorem clearly does not apply. The bound on the partition function just cannot be fulfilled.

Now consider the case of many-flavor fermions. Then, we have the product of all flavor determinants, which results in a real positive modulus plus an overall phase given by the sum of the complex phases of all flavors. For fermions of mass $M$, each phase is proportional to $M /|M|$, namely, to the mass's sign. This fact leads us to conclude, by using the same argument employed in the demonstration of the theorem, that the anomalous phases would cancel for an even number of flavors provided there is the same number of masses with opposite signs. This would make the resulting many-flavor determinant real and positive and would redeem the result of $P$ and $T$ invariance.

In our system, specifically, we have just seen in the previous section that in the low-temperature phase the dynamically generated electron masses present two opposite signs: $M= \pm\left|\epsilon_{0}\right|$; hence, the anomalous complex phases will cancel in compliance with the Vafa-Witten theorem.

The dynamical generation of masses and the associated occurrence of complex phases in the fermionic determinants, even though canceling when fully summed, are responsible for the onset of a nonvanishing valley current above the critical coupling $\alpha_{c}$ given by Eq. (50) and below 
the activation temperature $T^{*}$, which characterizes a QVHE.

This is the "center of gravity" of this work, the point where the dynamical generation of electron masses, obtained from the electron self-energy, meets the dynamical generation of a $P$ - and $T$-violating term in the vacuum polarization, for each flavor. By summing over the even number of flavors, the anomalous terms do cancel as a consequence of the fact that the masses are generated in pairs of opposite signs. This form of mass generation, despite ruling out a regular QHE, however, does imply a QVHE.

\section{APPENDIX B: NONRELATIVISTIC LIMIT}

Here, we investigate the small $v_{F} / c$ limit of the Dirac equation, assuming we are in the phase where the energy states $\epsilon_{n}^{( \pm)}$are present and give a mass to the electrons. Then, the Foldy-Wouthuysen transformation can be applied to the Dirac equation coupled to the pseudoelectromagnetic field. This result can be easily obtained from the corresponding transformation in QED4 [53], simply by constraining the matter to move only in the $x-y$ plane with $J_{z}=0$ (no current matter in the $z$ direction).

Using the Fermi velocity divided by the light velocity as an expansion parameter, the nonrelativistic limit of the Dirac equation in the lowest approximation yields the Pauli equation, which contains (a) the minimal coupling with the vector potential $\propto(\mathbf{p}-\mathbf{A})^{2}$, (b) an electron-spin interaction with the magnetic field $\propto(\boldsymbol{\sigma} \cdot \mathbf{B})$, and (c) the static Coulomb interaction $\propto(1 / r)$.

In the absence of a magnetic field, the second-order term in the expansion gives other interactions related to the electric field: (a) a Darwin interaction $\propto \rho(\mathbf{r})$ and (b) a spinorbit term which, taking $J_{z}=0$, reduces to a Rashba-like spin-orbit coupling. By applying an electric field in the $z$ direction, for instance, we obtain a spin-orbit coupling $\propto\left(\sigma_{x} p_{y}-\sigma_{y} p_{x}\right)$. It was recently shown that it is possible to generate quantum Hall states in the presence of a Rashba spin-orbit coupling and static interactions [54]. Since the spin-orbit coupling is included in the full electromagnetic interaction and this produces the QVHE, there could be a relation between the two effects. We shall explore this connection elsewhere.

[1] M. I. Katsnelson, K. S. Novoselov, and A. K. Geim, Chiral Tunnelling and the Klein Paradox in Graphene, Nat. Phys. 2, 620 (2006).

[2] M. I. Katsnelson, Zitterbewegung, Chirality, and Minimal Conductivity in Graphene, Eur. Phys. J. B 51, 157 (2006); J. Tworzydlo, B. Trauzettel, M. Titov, A. Rycerz, and C. W. J. Beenakker, Sub-Poissonian Shot Noise in Graphene, Phys. Rev. Lett. 96, 246802 (2006).
[3] D. Allor, T. D. Cohen, and D. A. McGady, Schwinger Mechanism and Graphene, Phys. Rev. D 78, 096009 (2008).

[4] K. S. Novoselov, A. K. Geim, S. V. Morozov, D. Jiang, M. I. Katsnelson, I. V. Grigorieva, S. V. Dubonos, and A. A. Firsov, Two-Dimensional Gas of Massless Dirac Fermions in Graphene, Nature (London) 438, 197 (2005).

[5] R. R. Nair, P. Blake, A. N. Grigorenko, K. S. Novoselov, T. J. Booth, T. Stauber, N. M. R. Peres, and A. K. Geim, Fine Structure Constant Defines Visual Transparency of Graphene, Science 320, 1308 (2008).

[6] H. C. Kao, M. Lewkowicz, and B. Rosenstein, Ballistic Transport, Chiral Anomaly, and Emergence of the Neutral Electron-Hole Plasma in Graphene, Phys. Rev. B 82, 035406 (2010); M. Lewkowicz, B. Rosenstein, and D. Nghiem, Two Distinct Ballistic Processes in Graphene at the Dirac Point, Phys. Rev. B 84, 115419 (2011).

[7] V. Juričić, O. Vafek, and I. F. Herbut, Conductivity of Interacting Massless Dirac Particles in Graphene: Collisionless Regime, Phys. Rev. B 82, 235402 (2010); I. F. Herbut, V. Juricic, and O. Vafek, Coulomb Interaction, Ripples, and the Minimal Conductivity of Graphene, Phys. Rev. Lett. 100, 046403 (2008).

[8] A. W. W. Ludwig, M. P. A. Fisher, R. Shankar, and G. Grinstein, Integer Quantum Hall Transition: An Alternative Approach and Exact Results, Phys. Rev. B 50, 7526 (1994).

[9] K. Ziegler, Minimal Conductivity of Graphene: Nonuniversal Values from the Kubo Formula, Phys. Rev. B 75, 233407 (2007); L. A. Falkovsky and S. S. Pershoguba, Optical Far-Infrared Properties of a Graphene Monolayer and Multilayer, Phys. Rev. B 76, 153410 (2007); T. Stauber, N. M. R. Peres, and A. K. Geim, Optical Conductivity of Graphene in the Visible Region of the Spectrum, Phys. Rev. B 78, 085432 (2008).

[10] M. O. Goerbig, Electronic Properties of Graphene in a Strong Magnetic Field, Rev. Mod. Phys. 83, 1193 (2011).

[11] A. H. Castro Neto, F. Guinea, N. M. R. Peres, K. S. Novoselov, and A. K. Geim, The Electronic Properties of Graphene, Rev. Mod. Phys. 81, 109 (2009).

[12] D. C. Elias, R. V. Gorbachev, A. S. Mayorov, S. V. Morozov, A. A. Zhukov, P. Blake, L. A. Ponomarenko, I. V. Grigorieva, K. S. Novoselov, F. Guinea, and A. K. Geim, Dirac Cones Reshaped by Interaction Effects in Suspended Graphene, Nat. Phys. 7, 701 (2011).

[13] M. A. H. Vozmediano and F. Guinea, Effect of Coulomb Interactions on the Physical Observables of Graphene, Phys. Scr. T146, 014015 (2012); F. de Juan, A. G. Grushin, and M. A. H. Vozmediano, Renormalization of Coulomb Interaction in Graphene: Determining Observable Quantities, Phys. Rev. B 82, 125409 (2010).

[14] X. Du, I. Skachko, A. Barker, and E. Y. Andrei, Approaching Ballistic Transport in Suspended Graphene, Nat. Nanotechnol. 3, 491 (2008).

[15] X. Du, I. Skachko, F. Duerr, A. Luican, and E. Y. Andrei, Fractional Quantum Hall Effect and Insulating Phase of Dirac Electrons in Graphene, Nature (London) 462, 192 (2009).

[16] K. 1. Bolotin, F. Ghahari, M. D. Shulman, H. L. Stormer, and P. Kim, Observation of the Fractional Quantum Hall Effect in Graphene, Nature (London) 462, 196 (2009). 
[17] F. Ghahari, Y. Zhao, P. Cadden-Zimansky, K. Bolotin, and P. Kim, Measurement of the $\nu=1 / 3$ Fractional Quantum Hall Energy Gap in Suspended Graphene, Phys. Rev. Lett. 106, 046801 (2011).

[18] C. R. Dean, A. F. Young, P. Cadden-Zimansky, L. Wang, H. Ren, K. Watanabe, T. Taniguchi, P. Kim, J. Hone, and K. L. Shepard, Multicomponent Fractional Quantum Hall Effect in Graphene, Nat. Phys. 7, 693 (2011).

[19] F. D. M. Haldane, Model for a Quantum Hall Effect without Landau Levels: Condensed-Matter Realization of the "Parity Anomaly”, Phys. Rev. Lett. 61, 2015 (1988).

[20] K. S. Novoselov, Z. Jiang, Y. Zhang, S. V. Morozov, H. L. Stormer, U. Zeitler, J. C. Maan, G. S. Boebinger, P. Kim, and A. K. Geim, Room-Temperature Quantum Hall Effect in Graphene, Science 315, 1379 (2007).

[21] C. L. Kane and E. J. Mele, Topological Order and the Quantum Spin Hall Effect, Phys. Rev. Lett. 95, 146802 (2005); Quantum Spin Hall Effect in Graphene, Phys. Rev. Lett. 95, 226801 (2005).

[22] E. G. Mishchenko, Minimal Conductivity in Graphene: Interaction Corrections and Ultraviolet Anomaly, Europhys. Lett. 83, 17005 (2008); D. E. Sheehy and J. Schmalian, Optical Transparency of Graphene as Determined by the Fine-Structure Constant, Phys. Rev. B 80, 193411 (2009); I. Sodemann and M. M. Fogler, Interaction Corrections to the Polarization Function of Graphene, Phys. Rev. B 86, 115408 (2012); D. N. Basov, M. M. Fogler, A. Lanzara, F. Wang, and Y. Zhang, Colloquium: Graphene Spectroscopy, Rev. Mod. Phys. 86, 959 (2014).

[23] I. F. Herbut and V. Mastropietro, Universal Conductivity of Graphene in the Ultrarelativistic Regime, Phys. Rev. B 87, 205445 (2013).

[24] G. D. Mahan, Many-Particle Physics (Plenum Press, New York, 1993).

[25] A. Kovner and B. Rosenstein, Kosterlitz-Thouless Mechanism of Two-Dimensional Superconductivity, Phys. Rev. B 42, 4748 (1990); N. Dorey and N. E. Mavromatos, QED and Two-Dimensional Superconductivity without Parity Violation, Nucl. Phys. B386, 614 (1992).

[26] E. C. Marino, Quantum Electrodynamics of Particles on a Plane and the Chern-Simons Theory, Nucl. Phys. B408, 551 (1993).

[27] R. L. P. G. do Amaral and E. C. Marino, Canonical Quantization of Theories Containing Fractional Powers of the d'Alembertian Operator, J. Phys. A 25, 5183 (1992).

[28] E. C. Marino, Complete Bosonization of the Dirac Fermion Field in $2+1$ Dimensions, Phys. Lett. B 263, 63 (1991).

[29] V. S. Alves, W. S. Elias, L. O. Nascimento, V. Juričić, and F. Peña, Chiral Symmetry Breaking in the Pseudo-Quantum Electrodynamics, Phys. Rev. D 87, 125002 (2013).

[30] A. Coste and M. Luscher, Parity Anomaly and FermionBoson Transmutation in 3-Dimensional Lattice QED, Nucl. Phys. B323, 631 (1989).

[31] M. D. Bernstein and T. Lee, Radiative Corrections to the Topological Mass in (2+1)-Dimensional Electrodynamics, Phys. Rev. D 32, 1020 (1985); S. Coleman and B. Hill, No More Corrections to the Topological Mass Term in $\mathbf{Q E D}_{3}$, Phys. Lett. 159B, 184 (1985).

[32] S. Teber, Electromagnetic Current Correlations in Reduced Quantum Electrodynamics, Phys. Rev. D 86, 025005
(2012); Two-Loop Fermion Self-Energy and Propagator in Reduced QED Q $_{3,2}$, Phys. Rev. D 89, 067702 (2014); A. V. Kotikov and S. Teber, Two-Loop Fermion Self-Energy in Reduced Quantum Electrodynamics and Application to the Ultrarelativistic Limit of Graphene, Phys. Rev. D 89, 065038 (2014); S. Teber and A. V. Kotikov, Interaction Corrections to the Minimal Conductivity of Graphene via Dimensional Regularization, Europhys. Lett. 107, 57001 (2014).

[33] L. Fritz, J. Schmalian, M. Muller, and S. Sachdev, Quantum Critical Transport in Clean Graphene, Phys. Rev. B 78, 085416 (2008).

[34] D. Xiao, W. Yao, and Q. Niu, Valley-Contrasting Physics in Graphene: Magnetic Moment and Topological Transport, Phys. Rev. Lett. 99, 236809 (2007).

[35] W. Yao, S. A. Yang, and Q. Niu, Edge States in Graphene: From Gapped Flat-Band to Gapless Chiral Modes, Phys. Rev. Lett. 102, 096801 (2009).

[36] N. Levy, S. A. Burke, K. L. Meaker, M. Panlasiqui, A. Zettl, F. Guinea, A. H. Castro Neto, and M. F. Crommie, StrainInduced Pseudo-Magnetic Fields Greater Than 300 Tesla in Graphene Nanobubbles, Science 329, 544 (2010).

[37] P. Ghaemi, J. Cayssol, D. N. Sheng, and A. Vishwanath, Fractional Topological Phases and Broken Time-Reversal Symmetry in Strained Graphene, Phys. Rev. Lett. 108, 266801 (2012).

[38] R. Nandkishore and L. Levitov, Quantum Anomalous Hall State in Bilayer Graphene, Phys. Rev. B 82, 115124 (2010).

[39] C. Vafa and E. Witten, Parity Conservation in Quantum Chromodynamics, Phys. Rev. Lett. 53, 535 (1984); Restrictions on Symmetry Breaking in Vector-Like Gauge Theories, Nucl. Phys. B234, 173 (1984); Eigenvalue Inequalities for Fermions in Gauge Theories, Commun. Math. Phys. 95, 257 (1984).

[40] For an excellent review on Schwinger-Dyson equations, see C. D. Roberts and A. G. Williams, Dyson-Schwinger Equations and Their Application to Hadronic Physics, Prog. Part. Nucl. Phys. 33, 477 (1994).

[41] R. D. Levine, Molecular Reaction Dynamics (Cambridge University Press, Cambridge, England, 2005).

[42] D. V. Khveshchenko, Ghost Excitonic Insulator Transition in Layered Graphite, Phys. Rev. Lett. 87, 246802 (2001); Magnetic-Field-Induced Insulating Behavior in Highly Oriented Pyrolitic Graphite, Phys. Rev. Lett. 87, 206401 (2001); D. V. Khveshchenko and H. Leal, Excitonic Instability in Layered Degenerate Semimetals, Nucl. Phys. B687, 323 (2004); D. V. Khveshchenko and W. F. Shively, Excitonic Pairing Between Nodal Fermions, Phys. Rev. B 73, 115104 (2006).

[43] E. V. Gorbar, V. P. Gusynin, V. A. Miransky, and I. A. Shovkovy, Magnetic Field Driven Metal-Insulator Phase Transition in Planar Systems, Phys. Rev. B 66, 045108 (2002); Fractal Structure of the Effective Action in (Quasi) Planar Models with Long-Range Interactions, Phys. Lett. A 313, 472 (2003).

[44] D. V. Khveshchenko, Massive Dirac Fermions in Single-Layer Graphene, J. Phys. Condens. Matter 21, 075303 (2009).

[45] H. Isobe and N. Nagaosa, Renormalization Group Study of Electromagnetic Interaction in Multi-Dirac-Node Systems, Phys. Rev. B 87, 205138 (2013). 
[46] M. Ezawa, Spin Valleytronics in Silicene: Quantum Spin Hall Quantum Anomalous Hall Insulators and Single-Valley Semimetals, Phys. Rev. B 87, 155415 (2013); ValleyPolarized Metals and Quantum Anomalous Hall Effect in Silicene, Phys. Rev. Lett. 109, 055502 (2012); Photoinduced Topological Phase Transition and a Single Dirac-Cone State in Silicene, Phys. Rev. Lett. 110, 026603 (2013).

[47] E. C. Marino, L. O. Nascimento, V. S. Alves, and C. Morais Smith, Unitarity of Theories Containing Fractional Powers of the d'Alembertian Operator, Phys. Rev. D 90, 105003 (2014).

[48] A. Rycerz, J. Tworzydlo, and C. W. J. Beenakker, Valley Filter and Valley Valve in Graphene, Nat. Phys. 3, 172 (2007).

[49] D. Gunlycke and C. T. White, Graphene Valley Filter Using a Line Defect, Phys. Rev. Lett. 106, 136806 (2011).
[50] T. Fujita, M. B. A. Jalil, and S. G. Tan, Valley Filter in Strain Engineered Graphene, Appl. Phys. Lett. 97, 043508 (2010).

[51] N. Myoung and G. Ihm, Gate-Tunable Valley-Filter Based on Suspended Graphene with Double Magnetic Barrier Structures, Curr. Appl. Phys. 14, 1455 (2014).

[52] J. Lahiri, Y. Lin, P. Bozkurt, I. I. Oleynik, and M. Batzill, An Extended Defect in Graphene as a Metallic Wire, Nat. Nanotechnol. 5, 326 (2010)

[53] J. D. Bjorken and S.D. Drell, Relativistic Quantum Mechanics (McGraw-Hill, College Park, MD, 1965).

[54] W. Beugeling, N. Goldman, and C. Morais Smith, Topological Phases in a Two-Dimensional Lattice: Magnetic Field Versus Spin-Orbit Coupling, Phys. Rev. B 86, 075118 (2012). 